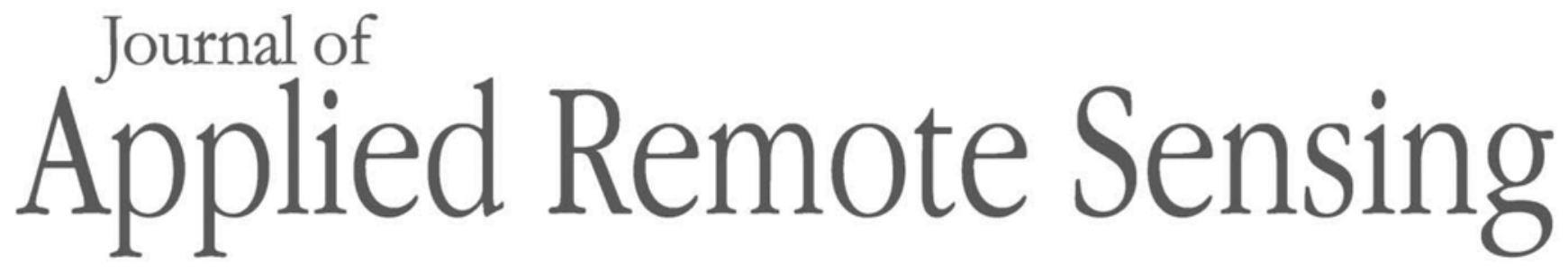

RemoteSensing.SPIEDigitalLibrary.org

\title{
Cotton growth modeling and assessment using unmanned aircraft system visual-band imagery
}

\author{
Tianxing Chu \\ Ruizhi Chen \\ Juan A. Landivar \\ Murilo M. Maeda \\ Chenghai Yang \\ Michael J. Starek
}




\title{
Cotton growth modeling and assessment using unmanned aircraft system visual-band imagery
}

\author{
Tianxing Chu, ${ }^{\text {a }}$ Ruizhi Chen, ${ }^{\text {b,* Juan A. Landivar, }},{ }^{c}$ Murilo M. Maeda, \\ Chenghai Yang, ${ }^{\mathrm{d}}$ and Michael J. Starek ${ }^{\mathrm{a}}$ \\ ${ }^{a}$ Texas A\&M University-Corpus Christi, Conrad Blucher Institute for Surveying and Science, \\ 6300 Ocean Drive, Corpus Christi, Texas 78412, United States \\ ${ }^{b}$ Wuhan University, State Key Laboratory of Information Engineering in Surveying, \\ Mapping and Remote Sensing, No. 129 Luoyu Road, Wuhan, Hubei 430079, China \\ ${ }^{\mathrm{c}}$ Texas A\&M AgriLife Research and Extension Center, 10345 TX-44, \\ Corpus Christi, Texas 78406, United States \\ dUSDA-Agricultural Research Service, 3103 F\&B Road, College Station, \\ Texas 77845, United States
}

\begin{abstract}
This paper explores the potential of using unmanned aircraft system (UAS)-based visible-band images to assess cotton growth. By applying the structure-from-motion algorithm, the cotton plant height $(\mathrm{ph})$ and canopy cover $(\mathrm{cc})$ information were retrieved from the point cloud-based digital surface models (DSMs) and orthomosaic images. Both UAS-based ph and cc follow a sigmoid growth pattern as confirmed by ground-based studies. By applying an empirical model that converts the cotton ph to cc, the estimated cc shows strong correlation $\left(R^{2}=0.990\right)$ with the observed cc. An attempt for modeling cotton yield was carried out using the ph and cc information obtained on June 26, 2015, the date when sigmoid growth curves for both ph and cc tended to decline in slope. In a cross-validation test, the correlation between the ground-measured yield and the estimated equivalent derived from the $\mathrm{ph}$ and/or $\mathrm{cc}$ was compared. Generally, combining ph and cc, the performance of the yield estimation is most comparable against the observed yield. On the other hand, the observed yield and cc-based estimation produce the second strongest correlation, regardless of the complexity of the models. (c) The Authors. Published by SPIE under a Creative Commons Attribution 3.0 Unported License. Distribution or reproduction of this work in whole or in part requires full attribution of the original publication, including its DOI. [DOI: 10.1117/1.JRS.10.036018]
\end{abstract}

Keywords: unmanned aircraft system; cotton yield; plant growth; regression; point cloud; orthomosaics.

Paper 16153 received Feb. 28, 2016; accepted for publication Aug. 2, 2016; published online Aug. 23, 2016.

\section{Introduction}

Monitoring crop growth has been realized to be of crucial importance for effective crop management. Factors of crop growth, such as plant height (ph), canopy cover (cc), canopy temperature, and water stress, are usually strong indicators for irrigation scheduling, harvest, fertilization, pesticide application, and production. ${ }^{1,2}$ Remote sensing has proven to be an appropriate technology accessing abundant, detailed, and quantitative crop information. ${ }^{3,4}$ For remotely monitoring the growth and health status, satellites provide well-tuned imagery products, however, their temporal and spatial limits sometimes tend to lag behind the requirement of crop growth monitoring, especially during the early stages of crop growth. In this regard, piloted aerial vehicles with portable remote sensors enable a complementary manner for monitoring a designated area ${ }^{5-7}$ Nevertheless, the use of an airborne sensing approach usually requires high user cost and professionalism, and may cause lengthy delivery of products and often limited temporal frequency. ${ }^{8}$ Moreover, airborne-based imagery is not capable of offering images

*Address all correspondence to: Ruizhi Chen, E-mail: ruizhi.chen@whu.edu.cn 
with sufficient detail to detect crop disease symptoms from individual plant leaves. ${ }^{9}$ As an alternative, ground-based sensing systems collect various in-situ data using equipment such as global positioning system, multispectral optical camera, ultrasonic ranging sensor, infrared radiometer, and temperature and relative humidity probes. ${ }^{10}$ The quality and performance of the data collected by ground-based sensing systems are usually satisfactory, ${ }^{11}$ although the data collection process is complex and time-consuming.

On the other hand, commercially available unmanned aircraft system (UAS) platforms are currently capable of carrying different types of remote sensors and have been recently explored for precision agriculture applications. ${ }^{12-14}$ The UAS platforms allow sensing crop growth with low flying altitude, flexible revisit frequency, and reasonable price. Recent UAS-relevant precision agriculture studies have used fixed-wing ${ }^{15,16}$ and multirotor ${ }^{17,18}$ platforms by carrying various types of equipment. ${ }^{8,19-22}$

Discrete ranging airborne LiDAR systems can provide multireturn capabilities and have been extensively utilized in forestry and vegetation for structure characterization and biomass estimation. ${ }^{23,24}$ However, over short cotton plants, which will be discussed in this study, the value of multireturn LiDAR is debatable because of limitations in range resolution. Range resolution in multireturn LiDAR refers to the minimum discrimination distance between consecutive returns (e.g., canopy top versus ground) along the slant path of the laser pulse. Range resolution is largely a function of the laser pulse length and response time of the receiver electronics for a given LiDAR system. The pulse widths of most commercially-available airborne LiDAR systems are too long to consistently capture more than one return in terms of observing a single cotton plant. For example, a 10-ns-pulse-length LiDAR system equates to 3-m "blind zone" for target discrimination with a single-channel detector. ${ }^{25}$ LiDAR systems with shorter pulse widths or full-waveform digitization may enhance the potential for effective cotton analysis, yet airborne LiDAR remains a relatively expensive method due to sensor costs and payload weight necessitating the use of larger aircraft. Although small UAS are now being equipped with lightweight, low-cost LiDAR sensors, the ranging characteristics of these sensors coupled with direct georeferencing solutions from the miniaturized onboard aiding technology limit performance. ${ }^{26}$ Point clouds produced from such systems are currently not equivalent to point cloud fidelity produced from expensive survey-grade airborne LiDAR systems although this gap will close as technology evolves.

As a sound and economical alternative to state-of-the-art LiDAR systems, new investigations have revealed great potentials in precision agriculture using visual-band consumer-grade cameras/red, green, and blue (RGB) cameras. ${ }^{27-29}$ As opposed to using costly LiDAR scanners, the structure-from-motion ( $\mathrm{SfM}$ ) techniques, developed by the photogrammetry community, have paved the way for utilizing low-cost visual-band cameras to access high-resolution three-dimensional (3-D) first-surface-return point cloud and plant profile information.

Recent studies, such as Refs. 16, 17, 19, 27-29, have investigated potentials for obtaining precise and reliable 3-D models of plants and achieved vegetation monitoring based on UAS imagery. Compared with previous works, the following contributions are addressed: (1) comprehensive cotton growth quantification was conducted in a test field from UAS imagery. While previous studies mainly focused on assessment of 3-D crop parameters from a single date flight campaign, comprehensive experimental campaigns with ultralow flight altitude were made in this study, enabling precise geospatial quantifications and full growth estimate over the life cycle of the cotton plants. (2) Cotton growth modeling and assessment using UAS visual-band imagery were introduced in this study. While related works primarily investigated trees and grain plants such as barley, UAS imagery-based cotton growth and vegetation monitoring potentials were rarely addressed. Furthermore, the relationship between cotton yield and plant growth parameters has not yet been sufficiently investigated, therefore, the potential of using plant growth parameters for yield prediction prior to maturation stage was examined in this study.

Therefore, a case study of comprehensive cotton growth modeling and assessment is presented in this paper. A lightweight UAS platform was setup and used to frequently fly over the designated cotton field. The study primarily focuses on the use of the low-cost UAS visible imagery and geospatial computing methods for estimating $\mathrm{cc}$ and ph information and analyzing their relationship with the cotton yield by testing regression models. The date when plant growth stabilizes was selected for developing and assessing the regression models. 


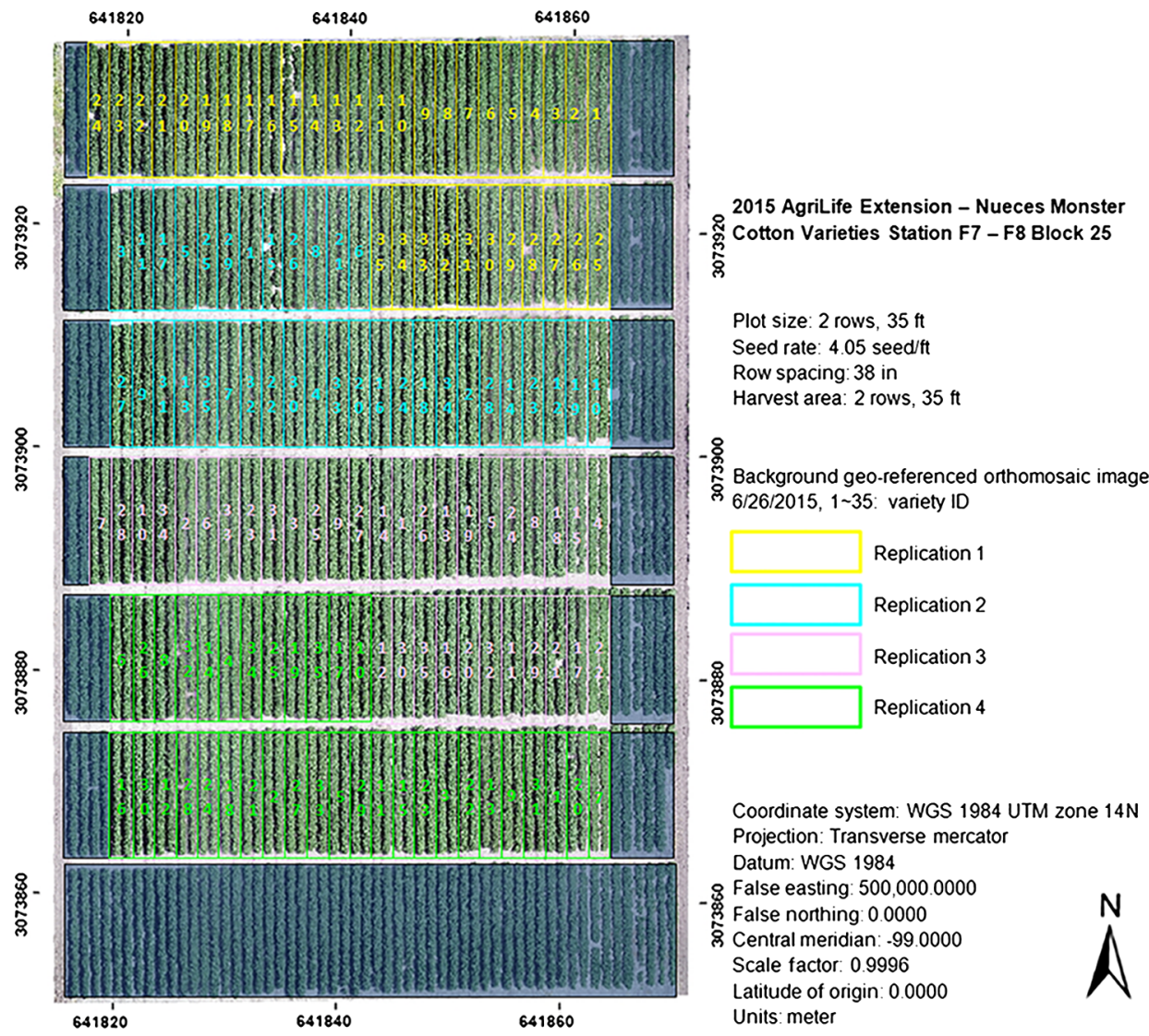

Fig. 1 Geographical illustration of the cotton study site.

\section{Material and Methods}

\subsection{Study Site}

A field trial was established at the Texas A\&M AgriLife Research and Extension Center $\left(27^{\circ} 46.948^{\prime} \mathrm{N}, 97^{\circ} 33.605^{\prime} \mathrm{W}\right)$ at Corpus Christi, Texas, during the summer of 2015 . The field was 85-m long and 54-m wide. Seeds of 35 cotton varieties were planted on April 1, 2015, in north-to-south oriented rows at a rate of 13 seeds $/ \mathrm{m}$. Each plot consisted of two rows that were $10.7-\mathrm{m}$ long, spaced at $0.96 \mathrm{~m}$. Each variety was replicated four times as indicated by different colors in Fig. 1. In the first replication, the variety numbers were arranged in ascending order, while in the latter replications, plots were arranged in a randomized complete block design. The border plots with dark blue color in Fig. 1 represented filler rows and were excluded from subsequent processing and analysis. Table 1 shows the cotton varieties and types planted in the study field. Cotton emergence was observed on April 6, 2015, and the cotton was harvested on August 17, 2015. Four ground control points (GCPs) were set up at the corners of the test field and used as geodetic benchmarks for image georeferencing purpose. The coordinates of the GCPs were measured using the Altus APS-3 receiver (Altus Positioning Systems, Torrance, California) in support of the TxDOT virtual reference station network, which enables instant access to centimeter-level positioning accuracy under the WGS-84 frame.

\subsection{Ground Data Collection}

Routine management practices such as fertility, disease prevention, and weed and insect control followed the guidelines provided by the Texas A\&M AgriLife Extension Service for the region. To determine population and plant density, stand counts were conducted on 0.0004 hectare on April 28, 2015, for each plot. All plots were harvested using a custom two-row cotton spindle 
Chu et al.: Cotton growth modeling and assessment using unmanned aircraft system visual-band imagery

Table 1 Cotton varieties and the types planted in the study field.

\begin{tabular}{|c|c|}
\hline Variety number & Variety type \\
\hline 1 & ST 4946 GLB2 \\
\hline 2 & PHY 333 WRF \\
\hline 3 & PHY 495 W3RF \\
\hline 4 & PHY 499 WRF \\
\hline 5 & PHY 312 WRF \\
\hline 6 & PHY 552 WRF \\
\hline 7 & PHY 444 WRF \\
\hline 8 & NG 3405 B2XF \\
\hline 9 & NG 3406 B2XF \\
\hline 10 & NG 5007 B2XF \\
\hline 11 & AMDG 7824 \\
\hline 12 & DG 3385 B2XF \\
\hline 13 & UA 103 \\
\hline 14 & UA 222 \\
\hline 15 & HQ $210 \mathrm{CT}$ \\
\hline 16 & ST 4747 GLB2 \\
\hline 17 & DP 1219 B2RF \\
\hline 18 & DP 1044 B2RF \\
\hline 19 & DP 1359 B2RF \\
\hline 20 & DP 1555 B2RF \\
\hline 21 & DP 1549 B2XF \\
\hline 22 & DP 1522 B2XF \\
\hline 23 & DP 1518 B2XF \\
\hline 24 & MON15R934XR \\
\hline 25 & ST 6182 GLT \\
\hline 26 & DP 1553 B2XF \\
\hline 27 & MON15R525 B2XF \\
\hline 28 & MON15R551 B2XF \\
\hline 29 & 12WSTR307-2 B2RF \\
\hline 30 & FM 2007 GLT \\
\hline 31 & CT15426 B2XF \\
\hline 32 & CT15545 B2XF \\
\hline 33 & CT15444 B2XF \\
\hline 34 & CT15425 B2XF \\
\hline 35 & CT15634 B2RF \\
\hline Filler & ST4946 GLB2 \\
\hline
\end{tabular}


picker model 9900 (Deere \& Company, Moline, Illinois) on August 17, 2015. This equipment was modified for small-plot research and allowed yield to be established on a per plot basis.

\subsection{Unmanned Aircraft System Platform and Imagery Data Collection}

The UAS platform used for this study was a Phantom 2 Vision+ multirotor copter (DJI, Shenzhen, Guangdong, China). Its integrated fisheye-lens camera provided 14 megapixel images with RGB channels. ${ }^{30}$ Its gimbal enabled stabilized nadir observation to the field during the flight experiments. Similar to the airborne photogrammetric technique, the UAS platform requires taking images with a high degree of spatial overlap for a favorable processing outcome. ${ }^{27}$ To select an appropriate flight strategy, the optimal image resolution, camera response time per shot, as well as the battery life were also taken into consideration. After balancing all these factors, it was determined that the UAS flew at an average height of $15 \mathrm{~m}$ with an average horizontal speed of $1 \mathrm{~m} / \mathrm{s}$, and the camera captured a shot once the UAS traveled $1 \mathrm{~m}$ horizontally.

Raw images were collected throughout the whole cotton growing and development phases from early April to late July 2015 for a total of 16 datasets. Specifically, during the first week after emergence, data were collected on a per-day basis for the purpose of monitoring the germination process. The routine flight experiment was conducted every week or every other week afterward, depending on the local weather conditions.

The flight time for each mission was $\sim 20$ min to cover the whole test field, and the nadirview images were taken to enable $\sim 70 \%$ along-track and $60 \%$ across-track overlap between the images. All flights were conducted around 12:00 PM local time to ensure the homogeneity of light intensity. To minimize human intervention and maintain consistency between different flight attempts, the camera settings (e.g., white balance, ISO rating, sharpness, and exposure parameters) were kept on automatic or default mode.

\subsection{Growth Information Generation}

At this stage, the goal is to extract cotton ph and cc estimates for each flight. Figure 2 shows the overall processing sketch using RGB images obtained from our lightweight UAS platform. Our study case first utilized the Pix4Dmapper Pro software (Pix4D SA, 1015 Lausanne, Switzerland) to generate initial mapping products. The software uses scale-invariant feature transform, ${ }^{31}$ or

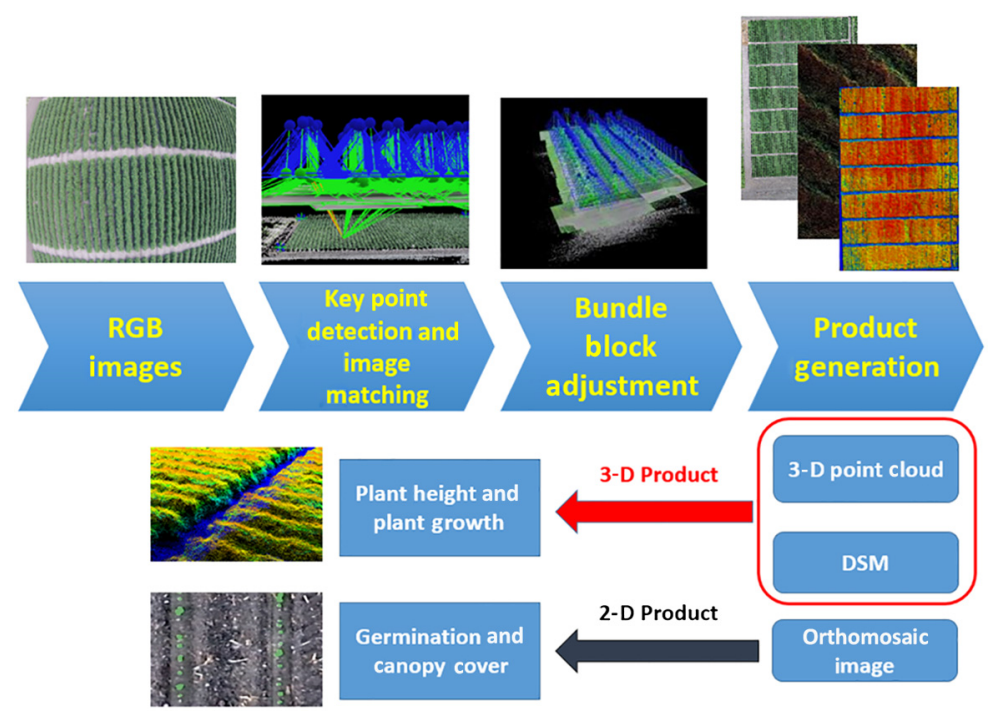

Fig. 2 Diagram of the cotton growth estimation using RGB images obtained from the lightweight UAS platform. 


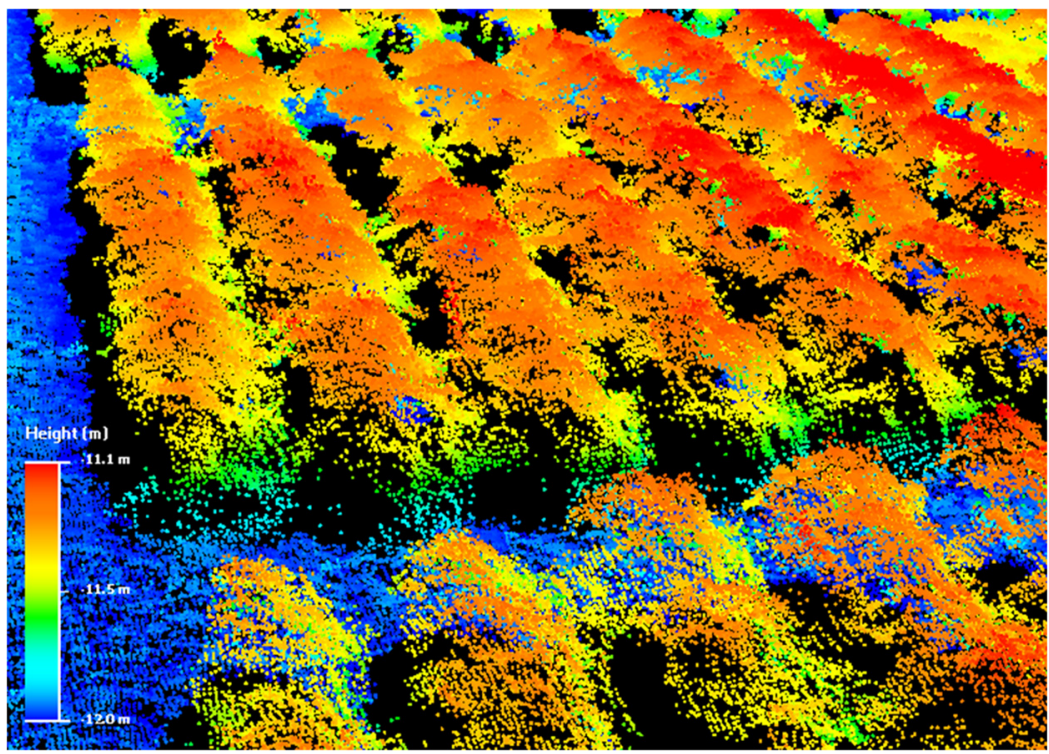

Fig. 3 An example of the point cloud data, based on the flight experiment carried out on June 26, 2015, focusing on a small part of the whole test field.

similar descriptor algorithm, to find key points and match a large set of images. It geometrically describes the projection between two corresponding points in a pair of images representing the same 3-D object. The GCPs were then loaded to the process to create georeferenced products. After importing the matched key points into the SfM algorithm, two-dimensional (2-D), and 3-D products (georeferenced orthomosaic images and 3-D point clouds) were generated. For generating 3-D models, SfM is an important stage to establish the correspondence between images and the reconstruction of 3-D objects to be studied. It requires multiple overlapped images as input to extract the 3-D point cloud given 2-D image common features. Detailed descriptions of the SfM algorithm and workflow can be found in Chapter 10 of Ref. 32. Similar to the Pix4Dmapper that was used in this research, the frequently implemented photogrammetry techniques can also be accessed in other equivalent commercially available software packages and open source solutions such as Agisoft PhotoScan Professional, ${ }^{33}$ Correlator3D UAV, ${ }^{34}$ insight $3 \mathrm{~d},{ }^{35}$ and VisualSFM. ${ }^{36}$

The orthomosaic images generated had an average file size of 410 megabytes. Figure 3 shows an example of the point cloud data, based on the flight experiment carried out on June 26, 2015, focusing on a small part of the whole test field. The legend shows negative height because the height was georeferenced to the WGS-84 reference ellipsoid. A $0.9-\mathrm{m}$ height difference is observable from the bare soil to the peak (highest point) of the cotton plants. In our study case, over 18 million points were generated in a point cloud file for the whole field for each flight, making the file size usually larger than 650 megabytes.

Cotton ph was estimated using the Quick Terrain Modeler 8.0.5 (Applied Imagery, Chevy Chase, Maryland) after loading the point clouds. In a first step, a bare soil-based digital surface model (DSM) (terrain elevation), $\mathrm{DSM}_{0}$, is supposed to be created by the point cloud dataset collected before cotton emergence. In this study, however, the first flight experiment was completed on April 7, 2015, the second day after cotton emergence was observed. In spite of that, its DSM was still considered the $\mathrm{DSM}_{0}$ due to the tiny and ignorable size of cotton hypocotyls observed by the UAS camera. The $\mathrm{DSM}_{0}$ was then subtracted from each subsequent digital surface model $\mathrm{DSM}_{i}$ after emergence, producing the cotton surface profile $\mathrm{CSP}_{i}$ at the $i$ 'th day after emergence (DAE). To allow an estimate of the uncertainty of height fields, the bare soil areas of the DSMs after emergence were compared against the $\mathrm{DSM}_{0}$. In general, the DSM uncertainty at the bare soil areas was $\sim 5 \mathrm{~cm}$ among all flight experiments in this study. It was mainly introduced by routine tractor plows, meteorology changes as well as image processing errors. 


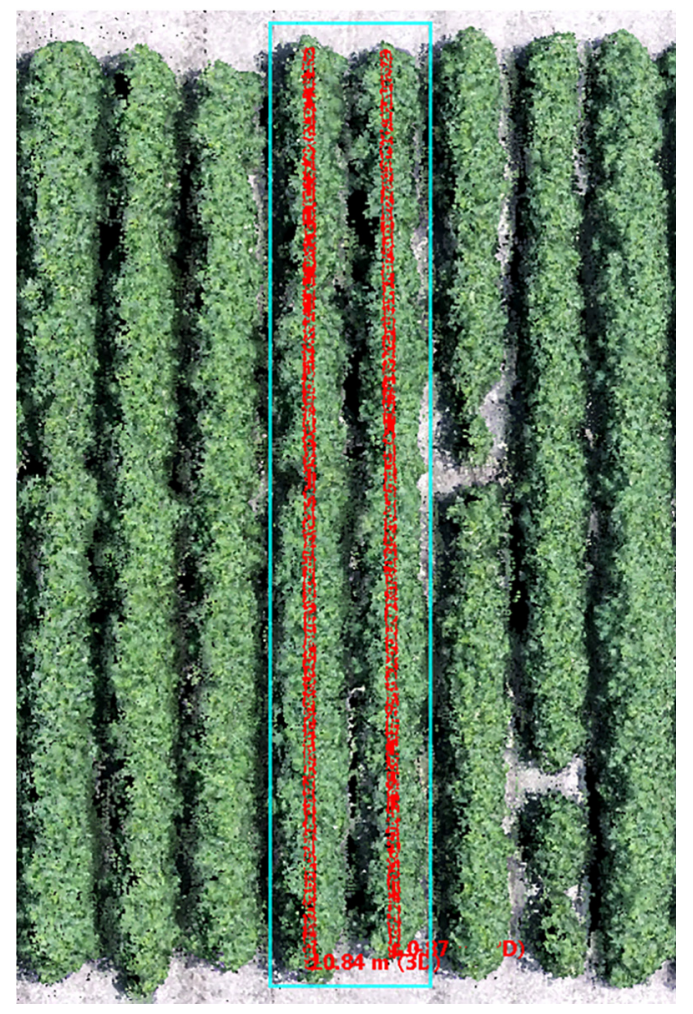

Fig. 4 Point cloud illustration of ph calculation for each cotton variety plot.

In a specific CSP model, the ph information of each variety was determined by averaging the height samples around the centerline of the cotton profile. More specifically in Fig. 4, the two rows in the cyan frame represent a variety plot, and the red dots depict the samples to be used for calculating the ph of this variety. The red dots were cloud points around the centerline of the cotton profile with $0.10-\mathrm{m}$ width at each row. The height was finally calculated by summing all the samples of these two rows and dividing by the sample number.

For assessing cc, the georeferenced orthomosaic images were loaded into ArcMap 10.3.1 platform (Esri, Redlands, California) for classification. The study area was classified into two categories (i.e., cotton canopy and bare soil). After manually training a few samples on each category, the software performed interactive supervised classification and cc was calculated as the proportion of the ground area covered by the vertical projection of crown perimeters, as described in Ref. 37.

\section{Results}

\subsection{Plant Height}

The UAS flight experiments ranged from April 7 to July 23, 2015, which covered the cotton growing and development cycle from emergence to early harvest stages. Due to the low flight altitude in this study case, the DSMs generated in Sec. 2.4 yielded $7.3 \mathrm{~mm} /$ pixel horizontal resolution. The ph and cc were estimated from April 12, 2015, (7 DAE) and the datasets collected previously were excluded because of the small size of the cotton seedlings during the early germination stage.

Cotton development follows a sigmoid curve pattern characterized by a slow growth rate during emergence, followed by a geometric increase in growth during leaf production, blooming and boll development phases, and slowing down and declining during maturation. ${ }^{38}$ Figure 5 illustrates the sigmoid regression curve of cotton ph against DAE based on the visible UAS imagery with SSE $=$ sum of squared errors, $R^{2}=$ coefficient of determination, adjusted $R^{2}=$ adjusted coefficient of determination, and RMSE $=$ root-mean-square errors. Each green 


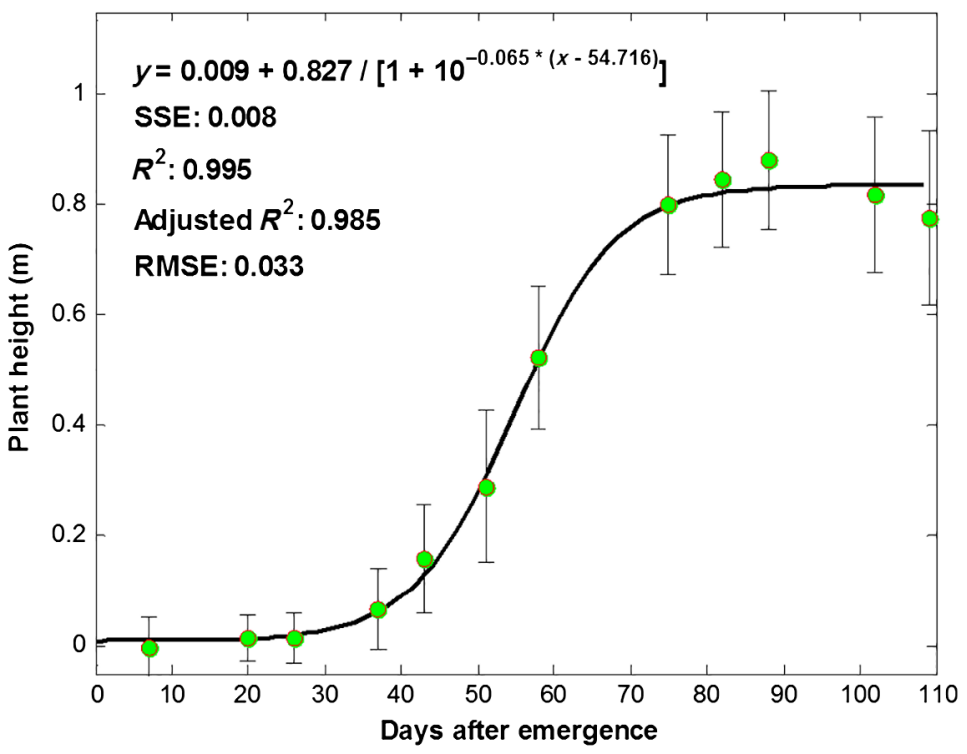

Fig. 5 Sigmoid regression of cotton ph against DAE based on UAS imagery.

dot represents the estimated ph on a specific data collection day by averaging the height statistics of all 35 varieties in 4 replications.

The cotton grew slowly before May 1, 2015, (26 DAE) and then exponentially until June 19, 2015 (75 DAE). The maximum crop height was observed on July 2, 2015 (88 DAE). The increased variation in crop height after $40 \mathrm{DAE}$ is likely caused by the phenotypic differences between the varieties.

\subsection{Canopy Cover}

In addition to the ph estimation, $\mathrm{cc}$ is also closely related to crop growth and development. To assess the cc, the orthomosaic images, which were created after applying the SfM algorithm, were loaded into the ArcMap engine. The camera settings were kept on automatic or default mode, and the image brightness values, for both bare soil and canopy, varied from day to day. Therefore, each orthomosaic image generated an individual set of training samples, representing either canopy or soil, and the interactive supervised classification strategy, specifically maximum likelihood classification using sample set, ${ }^{39}$ was performed to form the classified raster image. After making a raster-to-polygon conversion, $\mathrm{cc}$ was obtained by summing the area of all polygons over the investigated area. It is worth noting that the presence of weeds in the field may result in overestimation of cc. To eliminate the interference of the weeds, particularly during the early growing stages, polygons in between two adjacent rows were removed and excluded from the cc estimation. This process was performed by discriminating the 2-D coordinates of the centroid of the polygons, and any polygons that appeared off the cotton rows were filtered out.

The sigmoid curve in Fig. 6 depicts the cc regression against days after cotton emergence, confirming similar growth pattern as the ph. The cc calculated was based on the whole test field, rather than focusing on a specific area.

\subsection{Relationship Between Plant Height and Canopy Cover}

In this study, the ground measurements of cotton ph were not available, which disables a direct validation of the height model estimated from UAS imagery. Alternatively, an indirect method was conducted in this section to justify the UAS-based ph information.

As shown in Ref. 40, there is a linear relationship between ph and cc, which satisfies

$$
\mathrm{cc}=1.12 \times(\mathrm{ph} / \text { Row Spacing }) .
$$


Chu et al.: Cotton growth modeling and assessment using unmanned aircraft system visual-band imagery

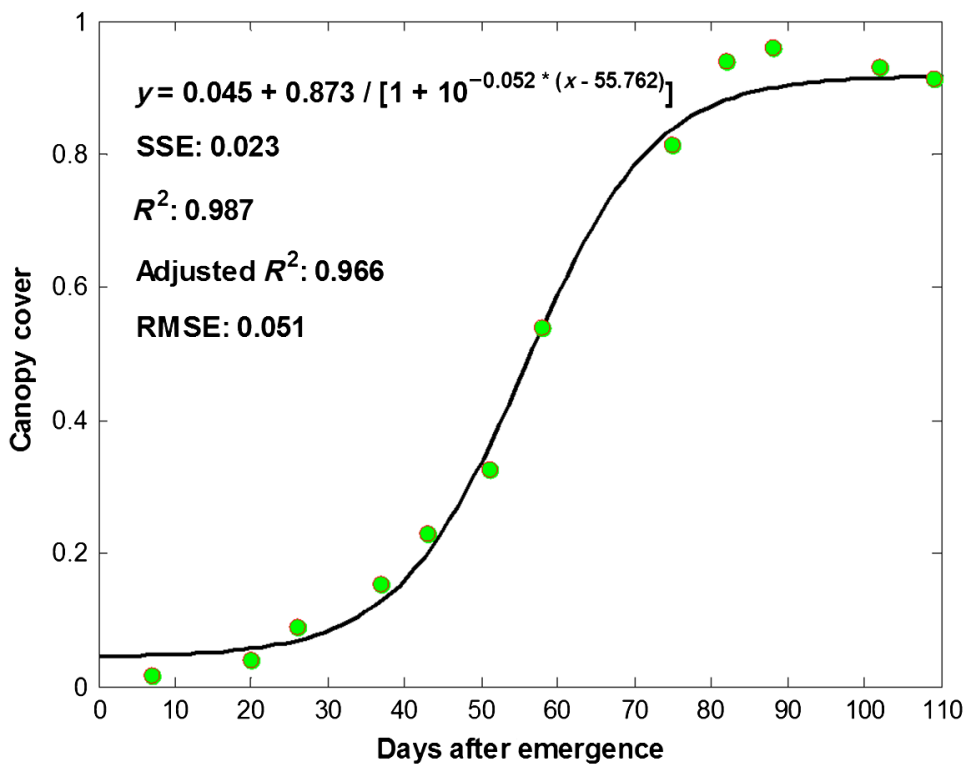

Fig. 6 Sigmoid regression of cc against DAE based on UAS imagery.

By applying Eq. (1), the estimated phs shown in Fig. 5 were converted to estimated ccs against DAE.

Comparison between observed cc derived from the orthomosaic image classification and estimated cc calculated using Eq. (1) is shown in Fig. 7. Results show a strong linear relationship between these two sets and demonstrate that the estimated and observed ccs coincide well with a coefficient of determination $R^{2}$ of 0.990 . This, in turn, proves the reliability of crop height estimated from UAS imagery. As for the validity of the observed cc, it will be addressed in a separate paper with an average accuracy of $88 \%$ achieved.

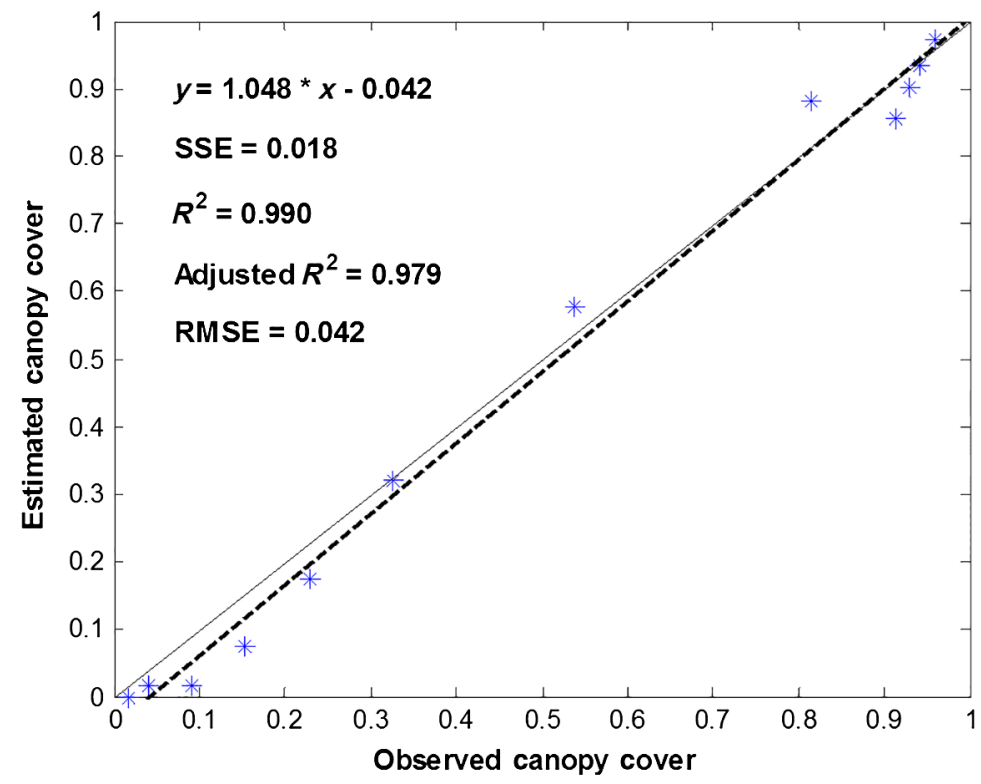

Fig. 7 Comparison between the observed cc derived from orthomosaic classification and estimated cc calculated using Eq. (1). The 1:1 relationship is represented by the solid line, and the dashed line depicts the linear regression between actual observed cc and estimated equivalent. 
Table 2 Regression models established for modeling cotton yield against the ph and/or cc in terms of linear, quadratic, and exponential fits.

\begin{tabular}{|c|c|c|c|c|}
\hline $\begin{array}{l}\text { Dependent } \\
\text { variable }\end{array}$ & $\begin{array}{l}\text { Independent } \\
\text { variable }\end{array}$ & Fit type & Regression model & \\
\hline \multirow[t]{12}{*}{$\begin{array}{l}\text { Yield } \\
\left(10^{3} \mathrm{~kg} / \mathrm{Ha}\right)\end{array}$} & $\mathrm{ph}(\mathrm{m}) \mathrm{cc}(\%)$ & Linear & $\mathrm{yld}=0.4951+2.93 \times \mathrm{ph}$ & (2) \\
\hline & & & $y l d=0.02329+4.841 \times c c$ & (3) \\
\hline & & & yld $=-0.3881+2.038 \times p h+2.693 \times c c$ & (4) \\
\hline & & Quadratic & $y l d=-2.978+11.43 \times p h-5.158 \times p^{2}$ & (5) \\
\hline & & & $y l d=5.452-13.33 \times c c+15.14 \times c^{2}$ & (6) \\
\hline & & & $\mathrm{yld}=1.162+19.69 \times \mathrm{ph}$ & \\
\hline & & & $-26.95 \times c c+0.7514 \times \mathrm{ph}^{2}$ & \\
\hline & & & $-31.84 \times \mathrm{ph} \times \mathrm{cc}+46.93 \times \mathrm{cc}^{2}$ & $(7)$ \\
\hline & & Exponential & $y l d=1.289 \times \exp (0.9852 \times p h)$ & (8) \\
\hline & & & $y l d=1.071 \times \exp (1.671 \times c c)$ & (9) \\
\hline & & & $\mathrm{yld}=1.36 \times \exp (0.7691 \times \mathrm{ph})$ & \\
\hline & & & $+0.002249 \times \exp (8.261 \times c c)$ & $(10)$ \\
\hline
\end{tabular}

\section{Yield Modeling and Discussion}

\subsection{Yield Modeling and Analysis}

In this paper, an investigation was conducted to model and assess the relationship of the cotton yield with the ph and/or cc on June 26, 2015, the approximate date of transition between rapid growth and crop maturation.

Three regression types (linear, quadratic, and exponential) were established for modeling the cotton yield (Table 2). The independent variables were ph and/or cc on June 26, 2015. The yield models were calibrated using all 35 cotton varieties (samples) of replications 2,3 , and 4 as shown in Fig. 1. Therefore, a total of 105 samples were used for model calibration Eqs. (2-10). In each sample, the plot yield, height, and cc information were calculated independently. On the other hand, the remaining 35 samples from replication 1 were used for model validation.

A comparison of the estimated yield against the observed yield for the 35 samples from replication 1 was made to evaluate the regression models. Correlations vary according to the models applied and Figs. 8-10 present the validation results estimating cotton yield using the models established in Table 2. Specifically, the estimated yields of subfigures $(\mathrm{a}-\mathrm{c})$ in Fig. 8 are derived from fitting Eqs. (2-4) in Table 2. Similarly, the estimated yields of subfigures in 


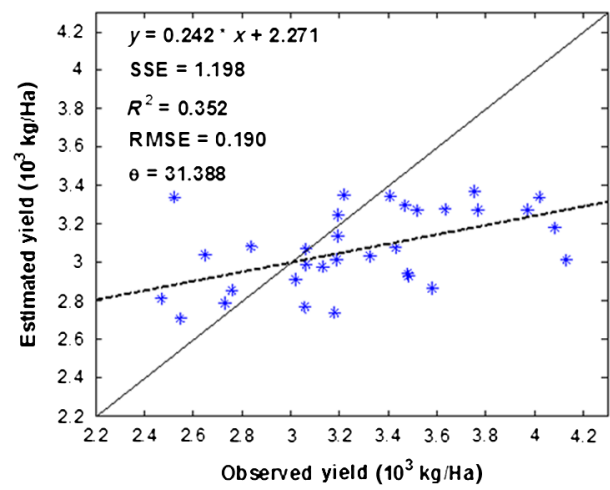

(a)

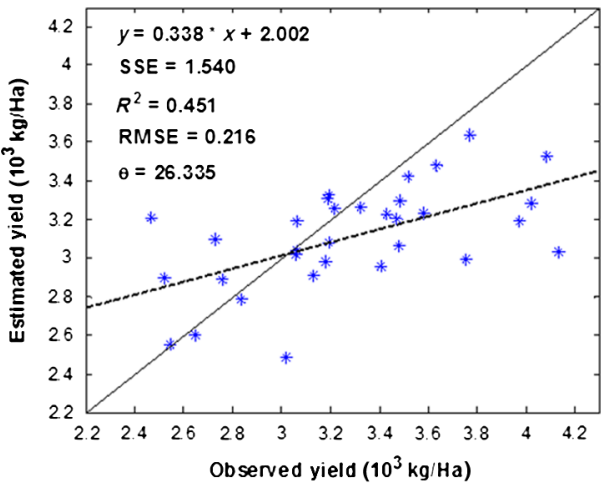

(b)

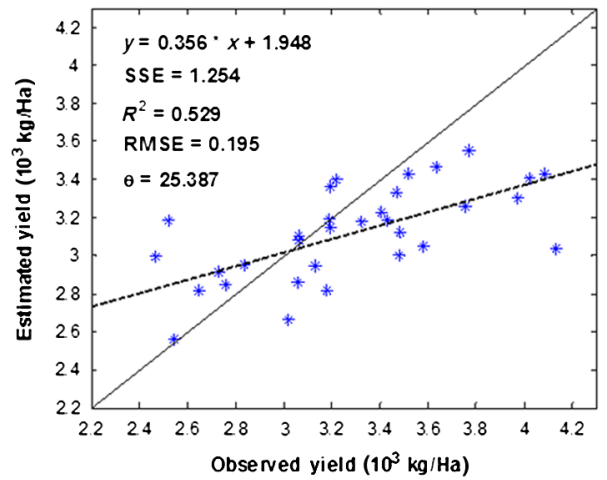

(c)

Fig. 8 Validation estimating cotton yield using the model Eqs. (2-4) established in Table 2. Estimated yields of subfigures (a), (b), and (c) are derived from fitting Eqs. (2), (3), and (4), respectively.

Figs. 9 and 10 are derived from Eqs. (5-7) and Eqs. (8-10), respectively. The blue asterisks in each subfigure depict 35 validation samples (varieties) in the first cotton replication. The dash lines illustrate the linear relationship between estimated and observed yield, while the solid lines indicate a 1:1 relationship. $\theta$ angle denotes the included angle between the dash line and solid line in units of degrees. A smaller $\theta$ angle indicates a smaller separation to the 1:1 line which represents the most ideal estimation-observation relationship.

Generally, the yield estimated by both ph and cc produces better linear results than those obtained by using only ph or cc, regardless of the fit type. In other words, Figs. 8(c), 9(c), and 10(c) outperform other solutions by using fitting Eqs. (4), (7), and (10), respectively, in terms of the SSE, $R^{2}$, RMSE, and $\theta$ angle. From the perspective of the fit type, all the models combining both ph and cc, i.e., Figs. 8(c), 9(c), and 10(c), generate similar correlation results as depicted in the corresponding subfigures. Likewise, the ph-only models, i.e., Figs. 8(a), 9(a), and 10 (a), are not sensitive to the applied fit types according to the in-figure evaluation statistics, yet generate the worst correlations. The performance of the models using only cc was found to be between the ph-only models and the ph/cc combination models. For example, Fig. 8(b) performs worse than Fig. 8(c), while better than Fig. 8(a). The same phenomenon also appears in exponential models. However, as an exception, Fig. 9(b) shows a slight improvement on the correlation statistics than Fig. 9(c) by estimating the yield using only $\mathrm{cc}$ and generates the least dispersed correlation results among quadratic Eqs. (5-7).

Therefore, according to the result analysis, cotton yield is found correlated to both ph and cc by establishing linear, quadratic, and exponential models as stated in Table 2 . These models generally agree with the expected behavior of larger yield at larger ph and cc. Different from previous studies on physiological progression of cotton growth and yield, these results provide a promising opportunity of predicting the yield of cotton when the rapid growth phase is about to finish before maturation and boll opening by considering the 2-D and 3-D growth statistics. Moving forward, upon these results, physiological rules might be acquired 


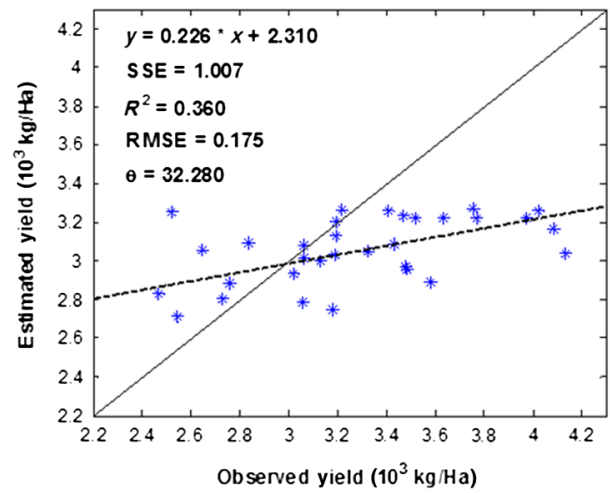

(a)

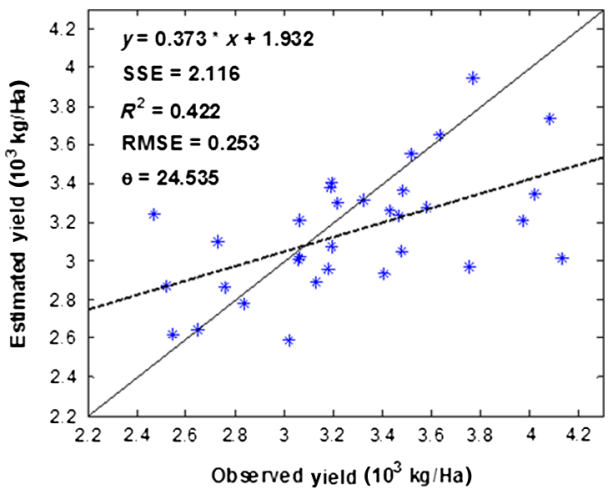

(b)

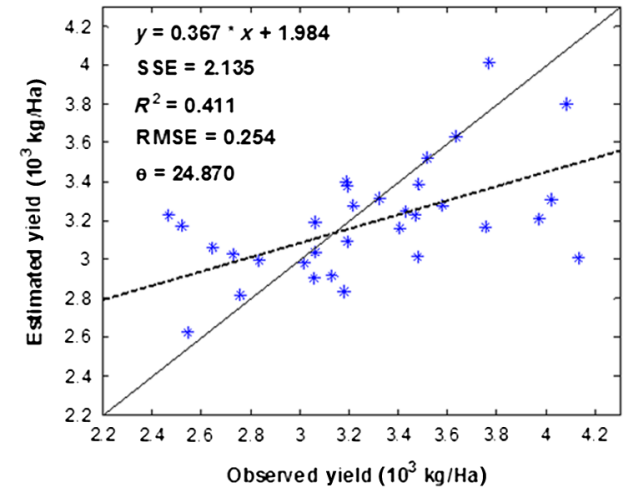

(c)

Fig. 9 Validation estimating cotton yield using the model Eqs. (5-7) established in Table 2. Estimated yields of subfigures (a), (b), and (c) are derived from fitting Eqs. (5), (6), and (7), respectively.

by agricultural producers to quantitatively manage and schedule plant care activities (such as irrigation, fertilization, and pest control) that, in turn, benefit healthy and robust growth of the cotton plants. To better understand the variability that may arise in estimating cotton growth and yield pattern to ensure the most efficient use of the concerns addressed above, producers or researchers are advised to arrange long-term observations under a multiyear scope. It is also worth noting that the estimation-observation validation lines, as shown in Figs. 8(c), 9(c), and 10 (c), are still $\sim 24$ deg off the 1:1 relationship, which makes most estimated yield lower than the observation. This phenomenon deserves further examination to enhance follow-up studies.

In this specific case, the independent variables used for estimating the cotton yield, i.e., the ph and cc, are all based on the image dataset collected on June 26, 2015, the end of the rapid increase in growth. The models established by using other dates may produce different results considering the nature of the cotton growth, and this possibility will be further investigated in a future study. Moreover, both the model training and validation phases used cotton samples of various varieties. This means the models proposed in this study ignore the differences of the growth characteristics of these varieties, thus a more delicate work needs to be carried out if aiming at estimating the yield of a specific variety.

\subsection{LiDAR-Derived Vegetation Metrics for Unmanned Aircraft System-Structure-From-Motion Point Cloud Data}

UAS-based SfM photogrammetry to derive 3-D point cloud data of crop structure, as performed here, represents an alternative to LiDAR. Although a comparison of airborne LiDAR versus UAS-SfM for crop monitoring is beyond the scope of discussion, it is important to emphasize some key differences between the methods to assess the potential of LiDAR-based metrics adapted to SfM point cloud data for progressing future results in this work. SfM relies on 


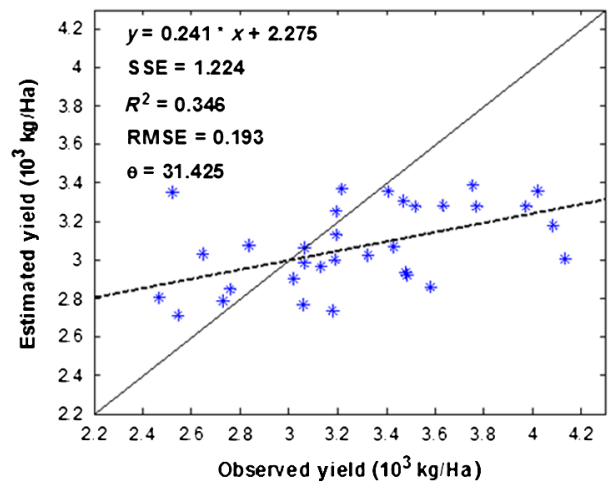

(a)

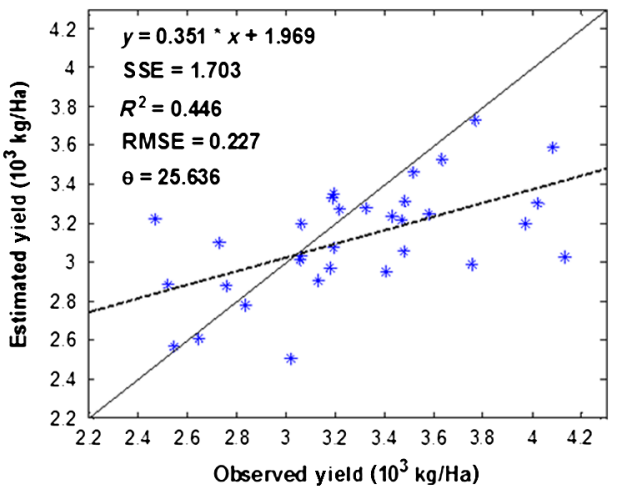

(b)

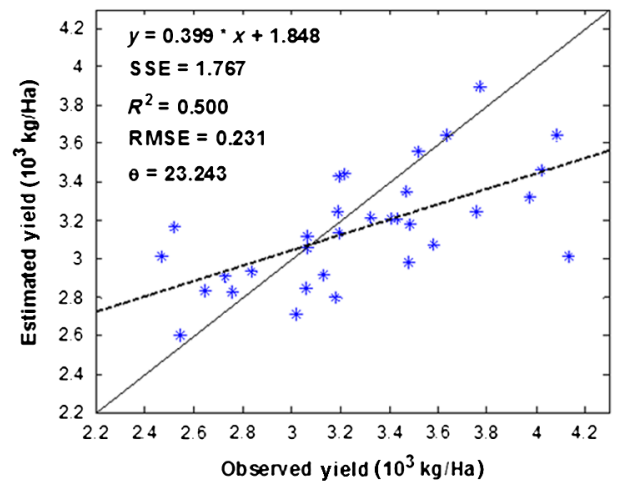

(c)

Fig. 10 Validation estimating cotton yield using the model Eqs. (8-10) established in Table 2. Estimated yields of subfigures (a), (b), and (c) are derived from fitting Eqs. (8), (9), and (10), respectively.

image-to-image pixel correspondence and collinearity to reconstruct the 3-D scene. As such, it generates what is called a first (or single) return point cloud, whereas modern discrete-return (or full-waveform) airborne LiDAR systems provide multireturn detection capability. ${ }^{41}$ This multireturn capability has rendered LiDAR being widely applied to forestry because it enables canopy and below canopy measurement. As mentioned in Sec. 1, however, the potential benefits of multireturn LiDAR data over short vegetation such as cotton is debatable due to limitations in the range resolution of many LiDAR systems. Another key difference in point cloud phenomenology between the two methods over vegetation stems from LiDAR being a pulsed ranging technique, whereas SfM is photogrammetric and susceptible to false parallax induced from moving vegetation between overlapping images (such as from wind). This can sometimes result in noisier point cloud data over vegetation dependent on weather conditions and vegetation structure. ${ }^{42}$ However, SfM computed from hyperspatial resolution imagery collected from a low flying UAS, as done here, can provide upward of two orders of magnitude increase in point density relative to traditional airborne LiDAR collected at higher altitudes above ground. This high point density enables noise to be easily smoothed and provides a high-definition point cloud for reconstructing vegetation structure.

In this work, SfM point cloud data were used to extract a single biophysical metric, mean ph, for which to assess cotton growth rates and predict yield performance. Results showed that mean ph values enabled high accuracy cc estimation validating SfM data integrity. Given the hyperspatial point density of the UAS-SfM approach, this opens the door for the application of a wide array of LiDAR vegetation metrics adapted to SfM point clouds for improving crop phenotyping and yield prediction. The literature provides numerous examples of LiDAR-based metrics for estimating biophysical attributes of vegetation, primarily from the forestry community. ${ }^{23,43-45}$ Multireturn LiDAR metrics often employ descriptive structure statistics calculated from height normalized LiDAR point clouds. These include selected height measures such as percentile of 
height, mean height, or max height; variability of height measures such as coefficient of variation; selected canopy return density measures; and selected cc measures such as cc above mean height. ${ }^{44}$ Full-waveform LiDAR metrics describe the radiometric and geometric attributes of the return waveforms over canopy. ${ }^{46}$ Waveform shape fitting methods are used to extract metrics such as height of median energy, standard deviation of pulse width, waveform distance, number of peaks, roughness of outermost canopy, and so on. ${ }^{44}$ With SfM being photogrammetric, full-waveform digitization is not plausible; however, several studies, such as Refs. 46 and 47, have shown the potential of using small-footprint, discrete-return LiDAR data to generate pseudowaveforms for reconstructing waveform composition over forested terrain. Such methods are well suited for adaption to the very high point density of the UAS-SfM point cloud for biophysical parameter estimation at the plant and plot level.

The aforementioned LiDAR metrics open an array of possibilities for enhancing UAS-SfM approaches to monitor crop performance such as cotton. Furthermore, by expanding the range of the input feature set, advanced machine learning approaches can be applied to progress the predictive performance for yield and growth estimation. Such exploration is currently being investigated in extension to the results presented here.

\section{Conclusions}

UAS platforms offer flexible, high labor efficiency, cost-effective, and nondestructive approaches to monitor crop growth and development with adequate resolution and revisit frequency. In this study, the feasibility to monitor and model life-cycle cotton growth using UAS imagery approach was examined. The imagery sensor used on the UAS was a commercial-grade fisheye RGB camera. A total of 12 image datasets, covering germination to early maturation phase, were used. By applying the SfM algorithm, individual images formed georeferenced orthomosaic images with dense 3-D point clouds. The ph and cc information, derived from the point clouds and orthomosaic images, follow the sigmoid growth curve as established by the ground-based measurements. Statistical analysis confirmed the reliability of UAS-based plant growth information by cross validating the ph and cc using an empirical model. Potential was found to predict the cotton yield using ph and cc obtained on June 26, 2015, the date when the sigmoid growth curve tended to decline in slope. However, the yield predicted by using both $\mathrm{ph}$ and $\mathrm{cc}$ is prone to slight underestimation, when compared to the groundmeasured yields. Moreover, the observed yield and cc-based estimation produces the second strongest correlation, regardless of the complexity of the models. On the contrary, the ph-only models on the same day, were found least correlated to the observed yield. This preliminary study seeks to help cotton producers acquire physiological rules for better management practices that are implemented today, with the ultimate goal of promoting sustainable cotton production.

Future studies involve collecting more datasets in a multiple-year scale, improving the yield estimation models by taking into account more parameters and the differences between varieties. In addition, future studies will also investigate data fusion from additional sensors, such as multispectral, hyperspectral, and thermal imaging sensors.

\section{Acknowledgments}

This work was partially funded by the Cotton Incorporated Project (No. 15-669TX) and the National Science Foundation Project (No. 1429518).

\section{References}

1. A. Srinivasan, Handbook of Precision Agriculture: Principles and Applications, Food Products Press, Binghamton, New York (2006).

2. M. A. Oliver, Geostatistical Applications for Precision Agriculture, Springer, Dordrecht, Netherlands (2010).

3. M. D. Johnson et al., "Crop yield forecasting on the Canadian Prairies by remotely sensed vegetation indices and machine learning methods," Agric. For. Meteorol. 218-219, 74-84 (2016). 
4. G. M. Richter et al., "Assessing on-farm productivity of Miscanthus crops by combining soil mapping, yield modelling and remote sensing," Biomass Bioenergy 85, 252-261 (2016).

5. C. Yang and W. C. Hoffmann, "Low-cost single-camera imaging system for aerial applicators," J. Appl. Remote Sens. 9(1), 096064 (2015).

6. C. Yang et al., "An airborne multispectral imaging system based on two consumer-grade cameras for agricultural remote sensing," Remote Sens. 6(6), 5257-5278 (2014).

7. $\mathrm{H}$. Li et al., "'Extended spectral angle mapping (ESAM)' for citrus greening disease detection using airborne hyperspectral imaging," Precis. Agric. 15(2), 162-183 (2013).

8. C. Zhang and J. M. Kovacs, "The application of small unmanned aerial systems for precision agriculture: a review," Precis. Agric. 13(6), 693-712 (2012).

9. F. Garcia-Ruiz et al., "Comparison of two aerial imaging platforms for identification of Huanglongbing-infected citrus trees," Comput. Electron. Agric. 91, 106-115 (2013).

10. R. Sui and J. A. Thomasson, "Ground-based sensing system for cotton nitrogen status determination," Trans. ASABE 49(6), 1983-1991 (2006).

11. Y. Huang et al., "Development and prospect of unmanned aerial vehicle technologies for agricultural production management," Int. J. Agric. Biol. Eng. 6(3), 1-10 (2013).

12. F. López-Granados et al., "Early season weed mapping in sunflower using UAV technology: variability of herbicide treatment maps against weed thresholds," Precis. Agric. 17, 183199 (2016).

13. C. M. Gevaert et al., "Generation of spectral-temporal response surfaces by combining multispectral satellite and hyperspectral UAV imagery for precision agriculture applications," IEEE J. Sel. Top. Appl. Earth Obs. Remote Sens. 8(6), 3140-3146 (2015).

14. J. Primicerio et al., "A flexible unmanned aerial vehicle for precision agriculture," Precis. Agric. 13(4), 517-523 (2012).

15. C. Lelong et al., "Assessment of unmanned aerial vehicles imagery for quantitative monitoring of wheat crop in small plots," Sensors 8(5), 3557-3585 (2008).

16. P. J. Zarco-Tejada et al., "Tree height quantification using very high resolution imagery acquired from an unmanned aerial vehicle (UAV) and automatic 3D photo-reconstruction methods," Eur. J. Agron. 55, 89-99 (2014).

17. J. Bendig et al., "Combining UAV-based plant height from crop surface models, visible, and near infrared vegetation indices for biomass monitoring in barley," Int. J. Appl. Earth Obs. Geoinf. 39, 79-87 (2015).

18. F.-J. Mesas-Carrascosa et al., "Assessing optimal flight parameters for generating accurate multispectral orthomosaicks by UAV to support site-specific crop management," Remote Sens. 7(10), 12793-12814 (2015).

19. H. Aasen et al., "Generating 3D hyperspectral information with lightweight UAV snapshot cameras for vegetation monitoring: from camera calibration to quality assurance," ISPRS J. Photogramm. Remote Sens. 108, 245-259 (2015).

20. P. J. Zarco-Tejada, V. González-Dugo, and J. A. J. Berni, "Fluorescence, temperature and narrow-band indices acquired from a UAV platform for water stress detection using a microhyperspectral imager and a thermal camera," Remote Sens. Environ. 117, 322-337 (2012).

21. F. Agüera, F. Carvajal, and M. Pérez, "Measuring sunflower nitrogen status from an unmanned aerial vehicle-based system and an on the ground device," Int. Arch. Photogram. Remote Sens. Spatial Inform. Sci. XXXVIII-1/C22, 33-37 (2011).

22. A. I. De Castro et al., "Detection of laurel wilt disease in avocado using low altitude aerial imaging," PLoS One 10(4), e0124642 (2015).

23. M. A. Wulder et al., "Lidar sampling for large-area forest characterization: a review," Remote Sens. Environ. 121, 196-209 (2012).

24. L. Wallace et al., "Development of a UAV-LiDAR system with application to forest inventory," Remote Sens. 4(6), 1519-1543 (2012).

25. K. C. Slatton et al., "Airborne laser swath mapping: achieving the resolution and accuracy required for geosurficial research," Geophys. Res. Lett. 34(23), L23S10 (2007).

26. R. A. Chisholm et al., "UAV LiDAR for below-canopy forest surveys," J. Unmanned Veh. Syst. 1, 61-68 (2013).

27. D. Gatziolis et al., "3D tree dimensionality assessment using photogrammetry and small unmanned aerial vehicles," PLoS One 10(9), e0137765 (2015). 
28. R. Díaz-Varela et al., "High-resolution airborne UAV imagery to assess olive tree crown parameters using 3D photo reconstruction: application in breeding trials," Remote Sens. 7(4), 4213-4232 (2015).

29. J. Torres-Sánchez et al., "High-throughput 3-D monitoring of agricultural-tree plantations with unmanned aerial vehicle (UAV) technology," PLoS One 10(6), e0130479 (2015).

30. DJI Phantom 2 Vision+, http://www.dji.com/product/phantom-2-vision-plus/feature (27 May 2016).

31. D. G. Lowe, "Distinctive image features from scale-invariant keypoints," Int. J. Comput. Vis. 60, 91-110 (2004).

32. R. Hartley and A. Zisserman, Multiple View Geometry in Computer Vision, 2nd ed., Cambridge University Press, Cambridge, UK (2004).

33. Agisoft PhotoScan, Professional Edition, Version 1.2.4, http://www.agisoft.com/ downloads/installer/ (27 May 2016).

34. Correlator3D UAV, http://www.simactive.com/en/correlator3d-uav/drone-photogrammetrysoftware/features (27 May 2016).

35. insight3d v0.3.2, http://insight3d.sourceforge.net/ (27 May 2016).

36. C. Wu, "VisualSFM: a visual structure from motion system," http://ccwu.me/vsfm/ (27 May 2016).

37. S. Jennings, N. Brown, and D. Sheil, "Assessing forest canopies and understorey illumination: canopy closure, canopy cover and other measures," Forestry 72(1), 59-74 (1999).

38. D. Zhao et al., "Growth and physiological responses of cotton (Gossypium hirsutum L.) to elevated carbon dioxide and ultraviolet-B radiation under controlled environmental conditions," Plant Cell Environ. 26(5), 771-782 (2003).

39. J. J. Settle and S. S. Briggs, "Fast maximum likelihood classification of remotely sensed imagery," Int. J. Remote Sens. 8(5), 723-734 (1987).

40. D. N. Baker, J. R. Lambert, and J. M. McKinion, "GOSSYM: a simulator of cotton crop growth and yield," Technical Bulletin No. 1089, South Carolina Agricultural Experiment Station, p. 134, Clemson University, Clemson, South Carolina (1983).

41. F. Leberl et al., "Point clouds: Lidar versus 3D vision," Photogramm. Eng. Remote Sens. 76(10), 1123-1134 (2010).

42. M. J. Starek et al., "Small-scale UAS for geoinformatics applications on an island campus," in IEEE Int. Conf. on Ubiquitous Positioning, Indoor Navigation and Location-Based Service (UPINLBS), pp. 120-127, Corpus Christi, Texas (2014).

43. S. C. Popescu, "Estimating biomass of individual pine trees using airborne Lidar," Biomass Bioenergy 31(9), 646-655 (2007).

44. L. Cao et al., "Using small-footprint discrete and full-waveform airborne LiDAR metrics to estimate total biomass and biomass components in subtropical forests," Remote Sens. 6(8), 7110-7135 (2014).

45. S. G. Zolkos, S. J. Goetz, and R. Dubayah, "A meta-analysis of terrestrial aboveground biomass estimation using Lidar remote sensing," Remote Sens. Environ. 128, 289-298 (2013).

46. T. Hermosilla et al., "Deriving pseudo-vertical waveforms from small-footprint fullwaveform LiDAR data," Remote Sens. Lett. 5(4), 332-341 (2014).

47. J. B. Blair and M. A. Hofton, "Modeling laser altimeter return waveforms over complex vegetation using high-resolution elevation data," Geophys. Res. Lett. 26(16), 2509-2512 (1999).

Tianxing Chu received his $\mathrm{PhD}$ in photogrammetry and remote sensing from Peking University, China. He is a postdoctoral research associate at Texas A\&M University-Corpus Christi (TAMUCC), Corpus Christi, Texas. His current research interests include applying remote sensing and geographical information system (GIS) techniques in the study of precision agriculture and unmanned aircraft system (UAS)-based remote sensing techniques for sustainable agriculture and precise vegetation monitoring, as well as ubiquitous navigation and positioning.

Ruizhi Chen received his MSc in computer science and engineering from Helsinki University of Technology and his PhD in geodesy from the University of Helsinki, both in Finland. He is 
a professor at Wuhan University, China. He has published 2 books, 5 book chapters, and more than 150 research articles. His current research interests include UAS-based remote sensing, precision agriculture, and mobile geospatial computing.

Juan A. Landivar received a doctorate in crop physiology from Mississippi State University in 1987. He is a professor and Texas A\&M AgriLife Research and Extension Center director in Weslaco and Corpus Christi, Texas. His research interests include developing improved cropping systems for better land and water management decisions, and computer models to evaluate crop management decisions.

Murilo M. Maeda received his MSc and PhD degrees in agronomy from TAMU in 2012 and 2015, respectively. He is an assistant research scientist at Texas A\&M AgriLife Research and Extension Center, Corpus Christi, Texas. His position focuses on the management of a cropping systems and remote sensing program for agricultural research applications and crop precision management.

Chenghai Yang received his $\mathrm{PhD}$ in agricultural engineering from the University of Idaho in 1994. He is an agricultural engineer with the U.S. Department of Agriculture, Agricultural Research Service's Aerial Application Technology Research Unit, College Station, Texas, USA. His current research is focused on the development and evaluation of remote sensing technologies for detecting and mapping crop pests for precision chemical applications.

Michael J. Starek received his $\mathrm{PhD}$ in civil engineering from the University of Florida. He is an assistant professor at the School of Engineering and Computing Sciences, TAMU-CC. He was formerly a National Research Council Postdoctoral Fellow of the US Army Research Office in affiliation with North Carolina State University. His research focuses on the application of emergent remote sensing and geomatics techniques for measurement and study of natural and built system dynamics. 